\title{
Strength of the spin-fluctuation-mediated pairing interaction in a high-temperature superconductor
}

\author{
T. Dahm ${ }^{1}$, V. Hinkov², S. V. Borisenko ${ }^{3}$, A. A. Kordyuk ${ }^{3}$, V. B. Zabolotnyy ${ }^{3}$, J. Fink ${ }^{3,4}$, B. Büchner ${ }^{3}$, \\ D. J. Scalapino ${ }^{5}$, W. Hanke ${ }^{6}$ and B. Keimer ${ }^{2 \star}$
}

\begin{abstract}
Theories based on the coupling between spin fluctuations and fermionic quasiparticles are among the leading contenders to explain the origin of high-temperature superconductivity, but estimates of the strength of this interaction differ widely'. Here, we analyse the charge- and spin-excitation spectra determined by angle-resolved photoemission and inelastic neutron scattering, respectively, on the same crystals of the high-temperature superconductor $\mathrm{YBa}_{2} \mathrm{Cu}_{3} \mathrm{O}_{6.6}$. We show that a self-consistent description of both spectra can be obtained by adjusting a single parameter, the spin-fermion coupling constant. In particular, we find a quantitative link between two spectral features that have been established as universal for the cuprates, namely high-energy spin excitations $^{2-7}$ and 'kinks' in the fermionic band dispersions along the nodal direction ${ }^{8-12}$. The superconducting transition temperature computed with this coupling constant exceeds $150 \mathrm{~K}$, demonstrating that spin fluctuations have sufficient strength to mediate high-temperature superconductivity.
\end{abstract}

Looking back at conventional superconductors, the most convincing demonstration of the electron-phonon interaction as the source of electron pairing was based on the quantitative correspondence between features in the electronic tunnelling conductance and the phonon spectrum measured by inelastic neutron scattering (INS; for reviews, see the articles by Scalapino, McMillan and Rowell in ref. 13). The rigorous comparison of fermionic and bosonic spectra was made possible by the Eliashberg theory, which enabled the tunnelling conductance to be derived from the experimentally determined phonon spectrum. Various difficulties have impeded a similar approach to the origin of high-temperature superconductivity. First, the $d$-wave pairing state found in these materials implies a strongly momentum-dependent pairing interaction. A more elaborate analysis based on data from momentum-resolved experimental techniques such as INS and angle-resolved photoemission spectroscopy (ARPES) is thus required. These methods, in turn, impose conflicting constraints on the materials. To avoid surface-related problems, most ARPES experiments have been carried out on the electrically neutral $\mathrm{BiO}$ cleavage plane in $\mathrm{Bi}_{2} \mathrm{Sr}_{2} \mathrm{Ca}_{n-1} \mathrm{Cu}_{n} \mathrm{O}_{2(n+2)+\delta}$ (ref. 8). However, as a consequence of electronic inhomogeneity, this family of materials exhibits broad INS spectra that greatly complicate a quantitative comparison with ARPES data ${ }^{7}$. Conversely, compounds with sharp spin excitations, including $\mathrm{YBa}_{2} \mathrm{Cu}_{3} \mathrm{O}_{6+x}$, have generated problematic ARPES spectra due to polar surfaces with charge distributions different from the bulk ${ }^{8}$. Finally, an analytically rigorous treatment of the spin-fluctuation-mediated pairing interaction is difficult, because small expansion parameters used in the traditional Eliashberg theory (such as the ratio of Debye and Fermi energies) are missing ${ }^{14}$. Because of these difficulties, widely different values have been quoted for the spin-fermion coupling constant ${ }^{1}$.

The analysis of $\mathrm{YBa}_{2} \mathrm{Cu}_{3} \mathrm{O}_{6.6}$ data reported here was made possible by recent advances on several fronts. First, INS experiments on this material now consistently yield high-quality spin-excitation spectra over a wide energy and momentum range ${ }^{2,4}$. Second, recent ARPES experiments on $\mathrm{YBa}_{2} \mathrm{Cu}_{3} \mathrm{O}_{6+x}$ (refs 11,12) have overcome problems related to polar surfaces and enabled the observation of superconducting gaps and band renormalization effects ('kinks') akin to those previously reported in La- and Bi-based cuprates ${ }^{8}$. Third, calculations based on the two-dimensional Hubbard model have demonstrated Fermi surfaces, single-particle spectral weights, antiferromagnetic spin correlations and $d_{x^{2}-y^{2}}$ pairing correlations in qualitative agreement with experimental measurements ${ }^{15-17}$. Numerically accurate solutions of this model can thus serve as a valuable guideline for a treatment of the spin-fluctuation interaction in the cuprates. This is the approach we take here.

Recent quantum Monte Carlo calculations of the twodimensional Hubbard model within the dynamical cluster approximation $^{17}$ for a realistic value of the bare $U / t=8$ and different doping levels ranging from underdoping to optimal doping have shown that the effective pairing interaction can be parameterized in terms of the numerically computed spin susceptibility $\chi(\mathbf{Q}, \Omega)$ in the form

$$
V_{\text {eff }}(\mathbf{Q}, \Omega)=\frac{3}{2} \bar{U}^{2} \chi(\mathbf{Q}, \Omega),
$$

where $\bar{U}$ is a renormalized coupling strength, and that this interaction generates reasonable values for the superconducting transition temperature $T_{\mathrm{c}}$. Here, we follow a similar strategy, but use $\chi(\mathbf{Q}, \Omega)$ determined by INS, on high-quality detwinned $\mathrm{YBa}_{2} \mathrm{Cu}_{3} \mathrm{O}_{6.6}$ single crystals described previously ${ }^{4}$. To serve as input for the numerical calculations, we have used an analytic form of $\operatorname{Im} \chi$ that provides an excellent description of the INS data (see Supplementary Information). Figure 1 shows a plot of this form in absolute units. In the superconducting state, the spin excitations exhibit the well-known 'hour glass' dispersion, with a neck at the wave vector $\mathbf{Q}=(\pi, \pi)$ characteristic of antiferromagnetism in the copper oxide planes and the 'resonance' energy $\Omega=38.5 \mathrm{meV}$. (We use a notation in which the lattice parameter $a$ and the reduced Planck constant $\hbar$ are set to unity. $\mathbf{Q}=(\pi, \pi)$ corresponds to $(0.5,0.5)$ in reciprocal lattice units, r.l.u.) The lower branch of

\footnotetext{
${ }^{1}$ Institute for Theoretical Physics, University of Tübingen, D-72076 Tübingen, Germany, ${ }^{2}$ Max-Planck-Institute for Solid State Research, D-70569 Stuttgart, Germany, ${ }^{3}$ IFW Dresden, PO Box 270116, D-01171 Dresden, Germany, ${ }^{4}$ BESSY, D-12489 Berlin, Germany, ${ }^{5}$ Department of Physics, University of California, Santa Barbara, California 93106-9530, USA, ${ }^{6}$ Institute for Theoretical Physics, University of Würzburg, D-97074 Würzburg, Germany. *e-mail: b.keimer@fkf.mpg.de.
} 


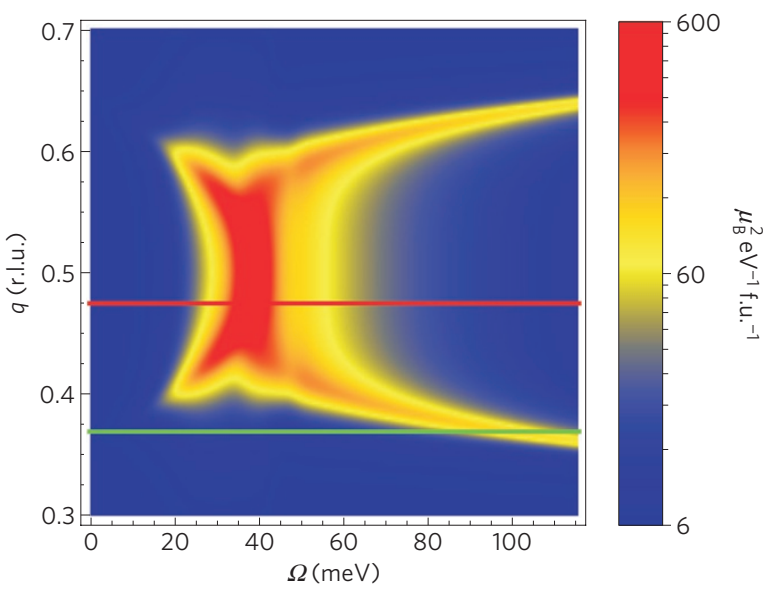

Figure 1 | Intensity of spin excitations along $Q=q(2 \pi, 2 \pi)$ resulting from numerical fits to the INS spectra of $\mathrm{YBa}_{2} \mathrm{Cu}_{3} \mathrm{O}_{6.6}$ at $\mathrm{T}=\mathbf{5} \mathrm{K}$. The maximum value corresponds to $600 \mu_{\mathrm{B}}^{2} \mathrm{eV}^{-1}$ f.u. ${ }^{-1}$ The green (red) line marks wave vectors connecting nodal (antinodal) regions on different Fermi surfaces (Fig. 2).

the hour glass seems to be influenced by materials-specific details. For instance, recent INS work on $\mathrm{La}_{2-x} \mathrm{Sr}_{x} \mathrm{CuO}_{4}$ indicates two characteristic energies ${ }^{5,6}$, rather than the single resonance found in $\mathrm{YBa}_{2} \mathrm{Cu}_{3} \mathrm{O}_{6+x}$. The upper branch of high-energy spin excitations, on the other hand, is common to all copper oxides thus far investigated by INS in this energy range $e^{2-7}$. Moreover, whereas the resonance in $\mathrm{YBa}_{2} \mathrm{Cu}_{3} \mathrm{O}_{6.6}$ disappears above $T_{\mathrm{c}}$ (ref. 4), the intensity of the spin excitations above $\Omega \sim 50 \mathrm{meV}$ is not noticeably affected by the superconducting transition and only decreases slowly on further heating ${ }^{2,4}$.

We extract the second parameter in equation (1), the coupling strength $\bar{U}$, from a combined analysis of the INS data parameterized in this way and the fermionic band dispersions observed by ARPES on the same crystals (see the Methods section). As noted before, bonding and antibonding combinations of electronic states on the two $\mathrm{Cu}-\mathrm{O}$ layers in the $\mathrm{YBa}_{2} \mathrm{Cu}_{3} \mathrm{O}_{6.6}$ unit cell give rise to two distinct Fermi surfaces (Fig. 2). The most prominent signature of many-body effects in the ARPES data, namely the 'kink' along the nodal direction (cut 1 in Fig. 3), is highlighted in Fig. 4, where the bonding band is singled out by a proper choice of excitation energy.

We now proceed to a quantitative analysis of the renormalization of the nodal band dispersion by spin fluctuations. Before describing the results, we take a look at the kinematics of spin-fluctuation scattering near the nodal points, where complications from the superconducting gap are absent. The spin fluctuations shown in Fig. 1 scatter electrons between bonding and antibonding bands, as indicated by factors in the INS (refs 2,4) and ARPES (refs 11,12) cross-sections. (Weak high-energy excitations corresponding to intraband scattering ${ }^{18}$ are neglected here.) An analysis of our numerical results below shows that the scattering probability for electrons near the nodal points is greatly enhanced when energy-momentum conservation enables interband scattering into opposite nodal regions (green arrow in Fig. 2). The INS data (green line in Fig. 1) reveal that this condition is satisfied by spin fluctuations of energy $\sim 80 \mathrm{meV}$ on the upper, universal, weakly temperature-dependent branch of the hour glass. At this characteristic energy in the temperature range studied here, we therefore expect a weakly temperature-dependent anomaly in the band dispersion, as experimentally observed.

A self-consistent numerical procedure with a single adjustable parameter, the coupling strength $\bar{U}$, was developed to quantitatively assess the influence of the spin-fluctuation interaction on the spectral function determined by ARPES (see the Methods section).

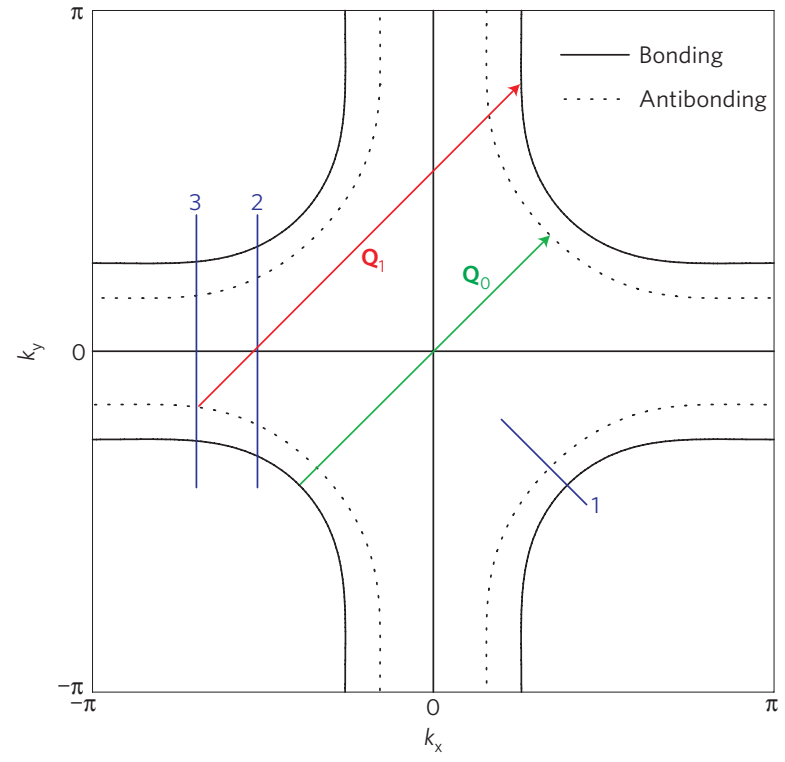

Figure 2 | Results of tight-binding fits of the Fermi surfaces determined by ARPES for the bonding and antibonding bands. The blue lines denote the three cuts along which experimental and theoretical spectral functions are compared (Fig. 3). The green and red arrows indicate spin-

fluctuation-mediated scattering processes discussed in the text.

Figure 4 shows that an excellent description of the nodal band dispersion over a wide energy range is obtained with $\bar{U}=1.59 \mathrm{eV}$, in rough agreement with values found in earlier calculations based on phenomenological models of the spin susceptibility ${ }^{1}$. In particular, both theoretical and experimental results show deviations from linear behaviour ('kink') for $\omega \geq 80 \mathrm{meV}$ (arrow in Fig. 4). The corresponding mass renormalization at the nodal point is $\operatorname{Re} Z_{\mathrm{A}}=3.7$.

Figure 3 shows a comparison of the calculated spectral weight to the ARPES intensity for all three cuts in Fig. 2. It is evident that the calculation yields an excellent description of the ARPES data set over the entire Brillouin zone without further fitting parameters. In particular, the low-intensity region ('dip') below the renormalized band in cut 3 can be understood as a consequence of coupling to the magnetic resonance at the neck of the hour-glass dispersion. As noted before ${ }^{1,19}$, the resonance wave vector (red lines in Figs 1 and 2) connects antinodal regions in bonding and antibonding bands, and the resonance and gap energies add up to the dip energy $\sim 65 \mathrm{meV}$. The only noticeable difference between the numerical and experimental data is the width of the momentum distribution curves, which is substantially larger in the ARPES data, presumably at least in part owing to residual surface inhomogeneities ${ }^{20}$.

Encouraged by the self-consistent description of INS and ARPES data, we proceed to a calculation of the critical temperature of the $d$-wave superconducting state arising from the exchange of spin fluctuations. A recent quantum Monte Carlo calculation of the two-dimensional Hubbard model within the dynamical cluster approximation has shown that a good estimate of $T_{c}$ can be obtained by using the same effective interaction as in the calculation of the self-energy ${ }^{17}$. For the set of parameters found above, the linearized gap equations (see the Methods section) yield the $d$-wave eigenvalue $\lambda_{d}=1.39$ in the normal state $(T=70 \mathrm{~K})$, corresponding to a transition temperature $T_{\mathrm{c}}=174 \mathrm{~K}$. In principle, the INS and ARPES spectra would now have to be remeasured at this higher temperature, the calculation repeated, and so on, until self-consistency is achieved. However, as the spectral weight rearrangement of spin excitations in this temperature range is largely confined to low excitation energies, our estimates for 


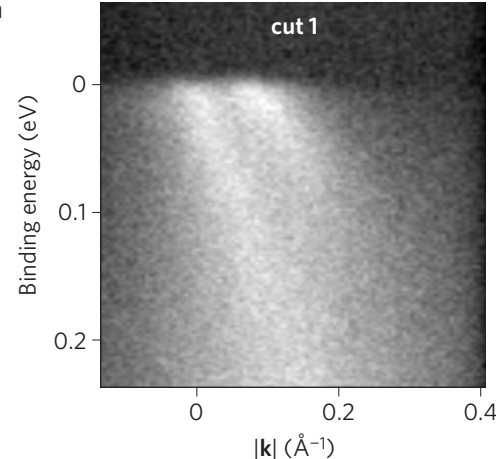

d

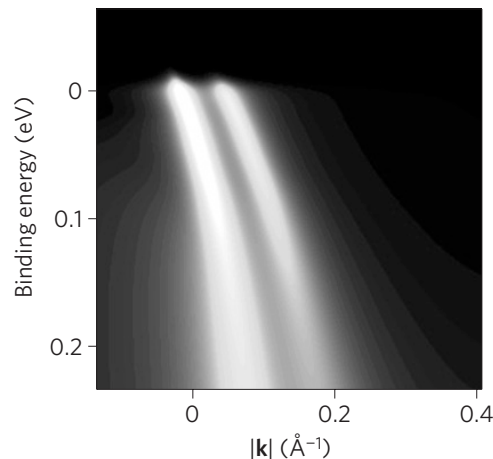

b

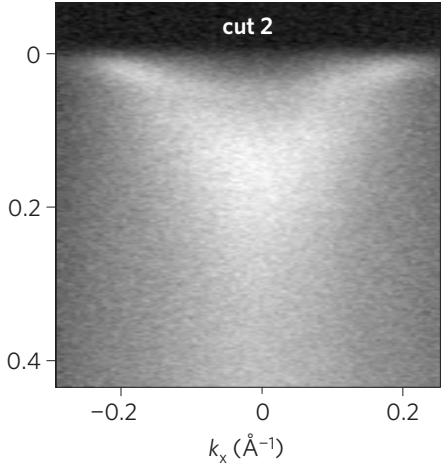

e

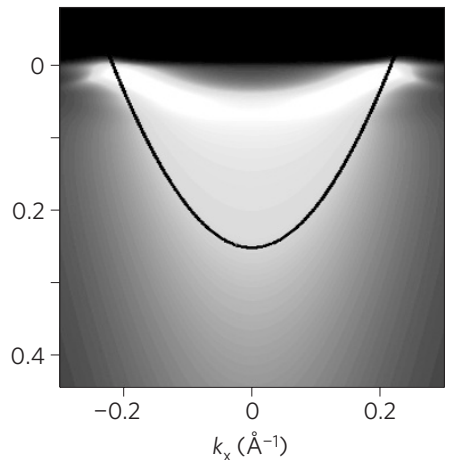

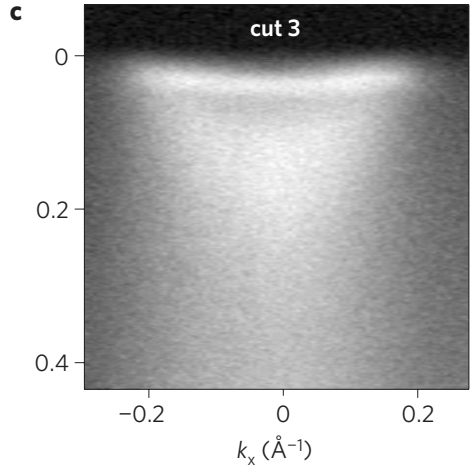

$\mathbf{f}$

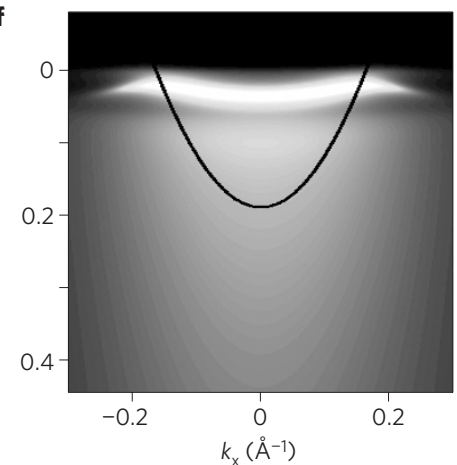

Figure 3 | Comparison of experimental and theoretical ARPES intensities. a-c, Experimental ARPES intensities at $30 \mathrm{~K}$ along the three cuts shown in Fig. 2, obtained with photon energies $60 \mathrm{eV}$ (cut 1) and $55 \mathrm{eV}$ (cuts 2 and 3). d-f, Theoretical spectral functions at $T=5 \mathrm{~K}$, multiplied by the Fermi function at $30 \mathrm{~K}$ (see the Methods section). Intensities in bonding and antibonding bands in $\mathbf{d}$ were multiplied by 0.75 and 0.25 , respectively, to take account of matrix element effects. The black lines in $\mathbf{e}$ and $\mathbf{f}$ show the unrenormalized antibonding band dispersion.

$\bar{U}$ and $T_{\mathrm{c}}$ are not expected to change substantially (see the Methods section). In this context, it is instructive to compare the eigenvalue at $T=70 \mathrm{~K}$ with the one obtained from the INS spectrum at $5 \mathrm{~K}$ that includes the 'resonance', $\lambda_{d}=1.49$. The enhanced eigenvalue implies that the redistribution of spectral weight of the spin excitations below $T_{\mathrm{c}}$ leads to an increase of the effective pairing strength. This lends support to an interpretation of the magnetic resonance and associated antinodal dip in terms of a superconductivity-induced feedback effect on the spin-fluctuation spectrum ${ }^{1}$. It is also consistent with the large $2 \Delta_{0} / k_{\mathrm{B}} T_{\mathrm{c}}$ ratio.

In summary, we have shown that data from two momentumresolved experimental probes of a cuprate superconductor can be related in a quantitative fashion, in close analogy to the traditional analysis of the electron-phonon interaction in conventional superconductors. Our analysis is in overall agreement with conclusions drawn from previous work based on phenomenological spin-excitation spectra and/or data from probes without momentum resolution ${ }^{1,19,21-23}$, and it resolves some problems that appeared in the context of these studies. In particular, models that attribute the nodal kink in ARPES either directly to the magnetic resonance ${ }^{24}$ or to incoherent scattering processes from a node into gapped states at the antinode $^{19}$ generally predict that the kink is strongly modified by the onset of superconductivity, whereas the experiments indicate at most a weak effect at $T_{\mathrm{c}}$ (ref. 10). The node-to-node interband scattering process mediated by weakly temperaturedependent, universal, incommensurate spin excitations we have identified provides a straightforward explanation of this observation. As the incommensurate spin excitations persist into the optimally doped ${ }^{5,7}$ and overdoped ${ }^{6}$ regimes, it also explains the persistence of both the kink and superconductivity at high doping, where feedback effects related to the magnetic

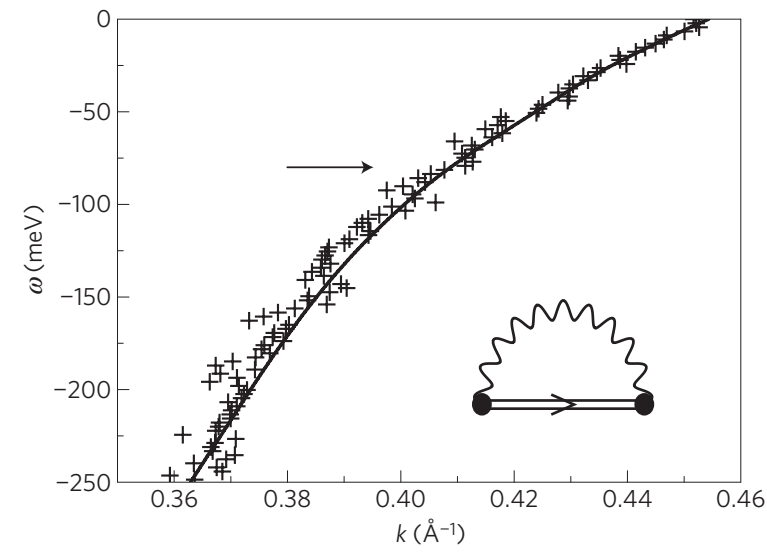

Figure 4 | Nodal dispersion of the ARPES data for the bonding band compared with the same nodal dispersion of the calculation. Crosses: ARPES data; solid line: calculation. Here, the coupling constant $\bar{U}$ was adjusted such that the slopes of the curves (renormalized Fermi velocity) match near zero energy. The inset shows a self-energy diagram for the exchange of spin fluctuations. The wiggly line represents the interaction given by equation (1), and the straight double line is the self-consistent single-particle Green's function.

resonance are progressively reduced ${ }^{22}$. There is thus no need to invoke phonon scattering at this level ${ }^{9}$. Although some contribution of phonons to the nodal kink cannot be ruled out ${ }^{25,26}$, recent work has shown that it is hard to obtain a quantitative description of the kink on the basis of the electron-phonon interaction alone ${ }^{19,27,28}$. An estimation of the influence of the electron-phonon interaction on our results can be found in Supplementary Information. 
It was previously shown ${ }^{29}$ that the change in the magnetic exchange energy between the normal and superconducting states is more than enough to account for the cuprate superconducting condensation energy. However, the crucial question of whether the exchange of magnetic spin fluctuations actually has the strength to give rise to high- $T_{\mathrm{c}}$ pairing, was not answered. Here, we have shown that this interaction can generate $d$-wave superconducting states with transition temperatures comparable to the maximum $T_{c}$ observed in the cuprates. In any given material, especially underdoped cuprates such as $\mathrm{YBa}_{2} \mathrm{Cu}_{3} \mathrm{O}_{6.6}$, a variety of effects not considered in our analysis can reduce the actual $T_{\mathrm{c}}$, including vertex corrections of the spin-fluctuation interaction ${ }^{14}$, phase fluctuations of the order parameter, competition with other types of order and pair breaking by phonons and impurities. It is also possible that phonons $s^{9,27,28}$ or higher-energy excitations ${ }^{30}$ contribute to the pairing interaction. However, our analysis indicates that the exchange of spin excitations already directly observed by INS is a major factor driving the high-temperature superconducting state in the cuprates.

\section{Methods}

The ARPES measurements were carried out on the same $\mathrm{YBa}_{2} \mathrm{Cu}_{3} \mathrm{O}_{6.6}$ crystals used for the INS experiment, thus avoiding systematic uncertainties invariably associated with measurements on different materials. The details of the ARPES experiments have been described elsewhere ${ }^{11,12}$. Usually, $\mathrm{YBa}_{2} \mathrm{Cu}_{3} \mathrm{O}_{6+x}$ single crystals cleave between the $\mathrm{Ba}-\mathrm{O}$ and $\mathrm{Cu}-\mathrm{O}$ chain layers, resulting in an effective overdoping of the $\mathrm{Cu}-\mathrm{O}$ layer closest to the surface ${ }^{11,12}$. A recent comprehensive study has revealed, however, that in some cases the ARPES spectra are dominated by a signal from the nominally doped $\mathrm{Cu}-\mathrm{O}$ plane ${ }^{12}$. Here, we present data taken on a particular spot on the surface after one such successful cleave (Figs 2,3). The strong many-body renormalization of the band structure typical for underdoped cuprates (Fig. 4) as well as the anisotropic superconducting gap (cuts 2 and 3 in Fig. 3 ) in the ARPES spectra demonstrates that contributions from the overdoped surface-related component are negligible. The superconducting component we observe corresponds to the nominal doping level, which we estimate from the size of the gap and the temperature evolution of the coherence peaks, which disappear above the bulk superconducting transition temperature ${ }^{12}$.

The self-consistent numerical calculation we have used is based on the self-energy diagram shown in Fig. 4. The Green's functions $G(\mathbf{k}, \omega)$ on antibonding (A) and bonding (B) bands can be written as ${ }^{15}$

$$
G_{\mathrm{A}, \mathrm{B}}(\mathbf{k}, \omega)=\frac{\omega Z_{\mathrm{A}, \mathrm{B}}+\tilde{\epsilon}_{k}^{\mathrm{A}, \mathrm{B}}}{\left(\omega Z_{\mathrm{A}, \mathrm{B}}\right)^{2}-\left(\tilde{\epsilon}_{k}^{\mathrm{A}, \mathrm{B}}\right)^{2}-\left(\operatorname{Re} Z_{\mathrm{A}, \mathrm{B}}(\mathbf{k}, \omega=0) \Delta_{k}\right)^{2}},
$$

where $\Delta_{k}$ is the superconducting gap, which we assume to be of the $d$-wave form $\Delta_{k}=\Delta_{0}\left(\cos k_{x}-\cos k_{y}\right) / 2$ with $\Delta_{0}=30 \mathrm{meV}$, and $\tilde{\epsilon}_{k}^{\mathrm{A}, \mathrm{B}}=\epsilon_{k}^{\mathrm{A}, \mathrm{B}}+\xi_{\mathrm{A}, \mathrm{B}}$ is the renormalized band structure. The unrenormalized band dispersions $\epsilon_{k}^{\mathrm{A}, \mathrm{B}}$ were derived from tight-binding fits to the ARPES Fermi surface in combination with extra information from band-structure calculations (see Supplementary Information). We have found that the results of our calculation are quite robust against modifications of $\epsilon_{k}^{\mathrm{A}, \mathrm{B}}$ (see Supplementary Information). Finally, $\omega Z_{\mathrm{A}, \mathrm{B}}(\mathbf{k}, \omega)=\omega-(1 / 2)\left(\Sigma_{\mathrm{A}, \mathrm{B}}(\mathbf{k}, \omega)-\Sigma_{\mathrm{A}, \mathrm{B}}^{*}(\mathbf{k},-\omega)\right)+i \Gamma_{\mathrm{el}}$ is the mass renormalization function and $\xi_{\mathrm{A}, \mathrm{B}}(\mathbf{k}, \omega)=(1 / 2)\left(\Sigma_{\mathrm{A}, \mathrm{B}}(\mathbf{k}, \omega)+\Sigma_{\mathrm{A}, \mathrm{B}}^{*}(\mathbf{k},-\omega)\right)$ is the energy shift function. Apart from an elastic scattering rate $\Gamma_{\mathrm{el}} \sim 30 \mathrm{meV}$, which accounts for impurity scattering, the mass renormalization function is determined by the imaginary part of the electron self-energy $\Sigma_{\mathrm{A}, \mathrm{B}}$, which can be written as

$$
\begin{aligned}
\operatorname{Im} \Sigma_{\mathrm{A}, \mathrm{B}}(\mathbf{k}, \omega)= & \frac{1}{\pi N} \sum_{\mathrm{Q}} \int_{-\infty}^{\infty} \mathrm{d} \Omega[n(\Omega)+f(\Omega-\omega)] \\
& \times \operatorname{Im} V_{\text {eff }}(\mathbf{Q}, \Omega) \operatorname{Im} G_{\mathrm{B}, \mathrm{A}}(\mathbf{k}-\mathbf{Q}, \omega-\Omega) .
\end{aligned}
$$

Here, $\sum_{Q}$ denotes a sum of the in-plane momenta over the full Brillouin zone, $n$ and $f$ are the Bose and Fermi functions, respectively, and $V_{\text {eff }}$ is the spin-fluctuation interaction equation (1). The real parts of $\Sigma_{\mathrm{A}, \mathrm{B}}$ that enter into equation (2) are obtained by Kramers-Kronig transformations. Note that the self-energy in the antibonding band is determined by the interaction with the bonding band and vice versa.

Together with equation (1), this defines a self-consistent system of equations with a single adjustable parameter, the coupling strength $\bar{U}$. Starting with non-interacting values for the Green's functions, these equations were solved iteratively until convergence was achieved. The renormalized band dispersion and spectral weight, $f(\omega) \operatorname{Im} G(\mathbf{k}, \omega)$, can then be compared with ARPES data.

The linearized gap equations

$$
\begin{aligned}
\lambda_{d} \operatorname{Im} \phi_{\mathrm{A}, \mathrm{B}}(\mathbf{k}, \omega)= & \frac{1}{\pi N} \sum_{k^{\prime}} \int_{-\infty}^{\infty} \mathrm{d} \omega^{\prime}\left[n\left(\omega-\omega^{\prime}\right)+f\left(-\omega^{\prime}\right)\right] \\
& \operatorname{Im} V_{\text {eff }}\left(\mathbf{k}-\mathbf{k}^{\prime}, \omega-\omega^{\prime}\right) \operatorname{Im}\left\{\frac{\phi_{\mathrm{B}, \mathrm{A}}\left(\mathbf{k}^{\prime}, \omega^{\prime}\right)}{\left(\omega^{\prime} Z_{\mathrm{B}, \mathrm{A}}\right)^{2}-\left(\tilde{\epsilon}_{k^{\prime}}^{B, A}\right)^{2}}\right\}
\end{aligned}
$$

were solved for the same set of parameters. Note that the INS data used for the calculations were taken at $T=5$ and $70 \mathrm{~K}$, whereas the ARPES data were taken at $30 \mathrm{~K}$. As the changes in the superconducting gap and INS spectrum between 5 and $30 \mathrm{~K}$ are negligible, we use the $5 \mathrm{~K}$ INS results along with the $30 \mathrm{~K}$ ARPES data to determine the coupling constant $\bar{U}$. As $T$ is raised further, the superconducting gap decreases, and there is a shift of $\operatorname{Im} \chi$ to lower frequencies. However, we expect that $\bar{U}$ is unchanged for the range of temperatures of interest, because it is determined by weakly $T$-dependent high-energy processes.

For discussions of the influence of a high-energy cutoff in $\chi(\mathbf{Q}, \Omega)$ and of a normal-state pseudogap on the results of the numerical calculations, see Supplementary Information.

Received 19 September 2008; accepted 12 December 2008; published online 18 January 2009

\section{References}

1. Eschrig, M. The effect of collective spin-1 excitations on electronic spectra in high- $T_{\mathrm{c}}$ superconductors. Adv. Phys. 55, 47-183 (2006).

2. Hayden, S. M., Mook, H. A., Dai, P., Perring, T. G. \& Dogan, F. The structure of the high-energy spin excitations in a high-transition-temperature superconductor. Nature 429, 531-534 (2004).

3. Tranquada, J. M. et al. Quantum magnetic excitations from stripes in copper oxide superconductors. Nature 429, 534-538 (2004).

4. Hinkov, V. et al. Spin dynamics in the pseudogap state of a high-temperature superconductor. Nature Phys. 3, 780-785 (2007).

5. Vignolle, B. et al. Two energy scales in the spin excitations of the high-temperature superconductor $\mathrm{La}_{2-x} \mathrm{Sr}_{x} \mathrm{CuO}_{4}$. Nature Phys. 3, 163-167 (2007)

6. Lipscombe, O. J., Hayden, S. M., Vignolle, B., McMorrow, D. F. \& Perring, T. G. Persistence of high-frequency spin fluctuations in overdoped superconducting $\mathrm{La}_{2-x} \mathrm{Sr}_{x} \mathrm{CuO}_{4}(x=0.22)$. Phys. Rev. Lett. 99, 067002 (2007).

7. Fauqué, B. et al. Dispersion of the odd magnetic resonant mode in nearly-optimally doped $\mathrm{Bi}_{2} \mathrm{Sr}_{2} \mathrm{CaCu}_{2} \mathrm{O}_{8+\delta}$. Phys. Rev. B 76, 214512 (2007).

8. Damascelli, A., Hussain, Z. \& Shen, Z.-X. Angle-resolved photoemission studies of the cuprate superconductors. Rev. Mod. Phys. 75, 473-541 (2003).

9. Lanzara, A. et al. Evidence for ubiquitous strong electron-phonon coupling in high-temperature superconductors. Nature 412, 510-514 (2001).

10. Kordyuk, A. A. et al. Constituents of the quasiparticle spectrum along the nodal direction of high- $T_{c}$ cuprates. Phys. Rev. Lett. 97, 017002 (2006).

11. Borisenko, S. V. et al. Kinks, nodal bilayer splitting and interband scattering in $\mathrm{YBa}_{2} \mathrm{Cu}_{3} \mathrm{O}_{6+x}$. Phys. Rev. Lett. 96, 117004 (2006).

12. Zabolotnyy, V. B. et al. Momentum and temperature dependence of renormalization effects in the high-temperature superconductor $\mathrm{YBa}_{2} \mathrm{Cu}_{3} \mathrm{O}_{7-\delta}$. Phys. Rev. B 76, 064519 (2007).

13. Parks, R. D. (ed.) Superconductivity Vol. 1 (Dekker, 1969).

14. Huang, Z. B., Hanke, W., Arrigoni, E. \& Chubukov, A. V. Renormalization of the electron-spin-fluctuation interaction in the $t-t^{\prime}-U$ Hubbard model. Phys. Rev. B 74, 184508 (2006).

15. Dahm, T. \& Tewordt, L. Physical quantities in nearly antiferromagnetic and superconducting states of the two-dimensional Hubbard model and comparison with cuprate superconductors. Phys. Rev. B $\mathbf{5 2}$ 1297-1308 (1995).

16. Aichhorn, M., Arrigoni, E., Potthoff, M. \& Hanke, W. Phase separation and competition of superconductivity and magnetism in the two-dimensional Hubbard model: From strong to weak coupling. Phys. Rev. B 76, 224509 (2007)

17. Maier, T. A., Macridin, A., Jarrell, M. \& Scalapino, D. J. Systematic analysis of a spin-susceptibility representation of the pairing interaction in the two-dimensional Hubbard model. Phys. Rev. B 76, 144516 (2007).

18. Pailhès, S. et al. Doping dependence of bilayer resonant spin excitations in (Y,Ca) $\mathrm{Ba}_{2} \mathrm{Cu}_{3} \mathrm{O}_{6+x}$. Phys. Rev. Lett. 96, 257001 (2006).

19. Chubukov, A. V. \& Norman, M. R. Dispersion anomalies in cuprate superconductors. Phys. Rev. B 70, 174505 (2004).

20. Graser, S., Hirschfeld, P. J. \& Scalapino, D. J. Local quasiparticle lifetimes in a $d$-wave superconductor. Phys. Rev. B 77, 184504 (2008).

21. Carbotte, J. P., Schachinger, E. \& Basov, D. N. Coupling strength of charge carriers to spin fluctuations in high-temperature superconductors. Nature 401, 354-356 (1999). 
22. Hwang, J., Timusk, T. \& Gu, G. D. High-transition-temperature superconductivity in the absence of the magnetic-resonance mode. Nature 427, 714-717 (2004).

23. Valla, T. Electronic interactions in strongly correlated systems: What is the 'glue' for high temperature superconductivity? Proc. SPIE 5932, 593203 (2005).

24. Manske, D., Eremin, I. \& Bennemann, K. H. Renormalization of the elementary excitations in hole- and electron-doped cuprates due to spin fluctuations. Phys. Rev. B 67, 134520 (2003).

25. Cuk, T. et al. Coupling of the $\mathrm{B}_{1 \mathrm{~g}}$ phonon to the antinodal electronic states of $\mathrm{Bi}_{2} \mathrm{Sr}_{2} \mathrm{Ca}_{0.92} \mathrm{Y}_{0.08} \mathrm{Cu}_{2} \mathrm{O}_{8+\delta}$. Phys. Rev. Lett. 93, 117003 (2004).

26. Reznik, D., Sangiovanni, G., Gunnarson, O. \& Devereaux, T. P. Photoemission kinks and phonons in cuprates. Nature 455, E6-E7 (2008).

27. Giustino, F., Cohen, M. L. \& Louie, S. G. Small phonon contribution to the photoemission kink in the copper oxide superconductors. Nature 452 975-978 (2008).

28. Heid, R., Bohnen, K.-P., Zeyher, R. \& Manske, D. Momentum dependence of the electron-phonon coupling and self-energy effects in superconducting $\mathrm{YBa}_{2} \mathrm{Cu}_{3} \mathrm{O}_{7}$ within the local density approximation. Phys. Rev. Lett. 100, 137001 (2008)
29. Woo, H. et al. Magnetic energy change available to superconducting condensation in optimally doped $\mathrm{YBa}_{2} \mathrm{Cu}_{3} \mathrm{O}_{6.95}$. Nature Phys. 2, 600-604 (2006).

30. Pasupathy, A. N. et al. Electronic origin of the inhomogeneous pairing interaction in the high- $T_{\mathrm{c}}$ superconductor $\mathrm{Bi}_{2} \mathrm{Sr}_{2} \mathrm{CaCu}_{2} \mathrm{O}_{8+\delta}$. Science 320, 196-201 (2008).

\section{Acknowledgements}

This project is part of the Forschergruppe FOR538 of the German Research Foundation. D.J.S. acknowledges the Center for Nanophase Material Sciences at Oak Ridge National Laboratory, US Department of Energy. We thank P. Bourges, A. Ivanov and D. Inosov for discussions.

\section{Additional information}

Supplementary Information accompanies this paper on www.nature.com/naturephysics. Reprints and permissions information is available online at http://npg.nature.com/ reprintsandpermissions. Correspondence and requests for materials should be addressed to B.K. 